\title{
Low-Molecular Weight Cow Colostrum Components in Functional Nutrition
}

\author{
A.I. Bozhkov*, E.G. Ivanov, Mohammad A.Y. Al Begai, Mohammad M.A. Alsardia and \\ N.I. Kurguzova
}

Research Institute of Biology V.N. Karazin National University of Kharkov, 4, Svobody Sq., 61022 Kharkov, Ukraine

\begin{abstract}
Background: The concept of functional nutrition has been actively developed in recent years. LMWCC contains biologically active compounds of natural origin and can be promising components of functional nutrition.

Methods: Wistar rats (intact, rats with $\mathrm{Cu}$-induced toxicosis and rats with $\mathrm{Cu}$-induced toxicosis, who received LMWCC per os in different doses of $0.01,0.05,0.1,1,2,3 \mathrm{mg} / 100 \mathrm{~g}$ of body weight of the animal). The effect of low-molecular weight components of cow colostrum (LMWCC) have been studied on the some physiological and biochemical parameters (body weight, temperature, ability to perform work, activity of aspartate aminotransferase, alanine aminotransferase, cholesterol, triacylglycerides, creatinine, albumin, gamma-glutamyltransferase and lipid hydroperoxides) in experimental animals with $\mathrm{Cu}$-induced toxicosis.
\end{abstract}

Results: It was found that LMWCC in small doses $(0.05-0.1 \mathrm{mg} / 100 \mathrm{~g}$ of weight) restored the growth rate, body temperature and partially working capacity in the test "swimming with a load" of animals with Cu-induced toxicosis. Restoration of physiological parameters in animals with $\mathrm{Cu}$-induced toxemia correlated with activation of the antioxidant enzyme glutathione peroxidase and a decrease in serum hydroperoxides in blood serum.

It was suggested that LMWCC can be promising components of functional nutrition for various toxicoses.

Conclusions: LMWCC has antioxidant properties in relatively small doses $(0.05-0.1 \mathrm{mg} / 100 \mathrm{~g}$ of weight). Restoration of the physiological characteristics of animals with $\mathrm{Cu}$-induced toxicosis correlates with the elimination of oxidative stress. Large doses of $1 \mathrm{mg} / 100 \mathrm{~g}$ of body weight and more manifest pro-oxidant properties.

Keywords: Pro-antioxidant system, Cu-induced toxicosis, liver.

\section{INTRODUCTION}

One of the priority areas of biomedical sciences is the development of the concept of functional nutrition $[1,2]$. The biological effect of functional nutrition can be achieved in the following cases: 1 - nutrition components contain natural biologically active compounds; 2 - a reasonable diet plan (dose, interval and timing of intake); 3 - accounting for the features of the body's functional systems (quantitative and qualitative "deviations" from homeostatic indices).

Only fundamental study of the mechanisms of action of nutrition components on the body can allow developing a single concept of functional nutrition. Components of cow colostrum can be considered as a promising substance in functional nutrition. Cow colostrum contains 3-5 times more protein compared to milk, rich in hormones, vitamins and, in particular, vitamin $A$, contains a wide range of cytokines, and biologically active metabolites [3-5]. All components of the colostrum can be divided into at least 3 groups: 1 factors regulating the immune response (in particular -

*Address correspondence to this author at the Research Institute of Biology V.N. Karazin National University of Kharkov, 4, Svobody Sq., 61022 Kharkov, Ukraine; E-mail: bozhkov@univer.kharkov.ua the transfer factor, cytokines); 2. - growth factors (insulin-like growth factors, TGF- $\alpha$, TGF- $\beta$, growth factor of fibroblasts, etc.); 3 - factors of the regulation of metabolism (leptin, insulin, cAMP, etc.) [6-8].

However, it can be noted a number of restrictions on the use of colostrum as a food product. Firstly, the colostrum contains a number of high molecular weight proteins that have allergic properties. Secondly, the colostrum composition changes very quickly during storage. The high content of biologically active compounds in the product can also cause negative effects.

We hypothesis that the isolation of individual biologically active compounds from the colostrum, the development of ways to stabilize and store these compounds can circumvent these limitations. The low molecular weight colostrum components were obtained in the present work, their effect on physiological and some biochemical indices of experimental animals with an increased contain of copper ions in the body were studied.

\section{MATERIALS AND METHODS}

All procedures with animals were carried out in compliance with bioethical principles [9] taking into

๑๐ 2017 Lifescience Global 
account the circadian rhythms of formation of biological responses. For this animal always received the food in the same time, were maintained under the standard conditions and all manipulation were carried out at the same time before feeding.

The experiments were carried out for 3 months male rats of the Wistar line. All animals were divided into three experimental groups. In each experimental group there were 9-12 animals.

The first group was the intact control, the animals were kept in standard vivarium conditions. The second group is animals with $\mathrm{Cu}$-induced toxicosis. To do this, they intraperitoneally injected an aqueous solution of copper sulphate at a dose of $1 \mathrm{mg} / 100 \mathrm{~g}$ of body weight three times at intervals of 48 hours between administrations. 24 hours after the administration of copper sulphate, the animals were lulled with ether anesthesia and taken into an experiment. The third group was the animals that were administered sulphate copper as well as the animals of the second group, and 24 hours later, low molecular weight components of the colostrum (LMWCC) were administered to them daily for 3 days. After 24 hours after the last administration of LMWCC, the animals were taken into the experiment.

The animals of this group were divided into several groups that received LMWCC in different doses: 0.01 $\mathrm{mg} / 100 \mathrm{~g}$ of body weight, $0.05 \mathrm{mg} / 100 \mathrm{~g}$ of body weight, $0.1 \mathrm{mg} / 100 \mathrm{~g}$ of body weight, $1 \mathrm{mg} / 100 \mathrm{~g}$ of body weight, $2 \mathrm{mg} / 100 \mathrm{~g}$ of body weight, $3 \mathrm{mg} / 100 \mathrm{~g}$ of body weight.
The body weight of the animals was determined from 1 to 15 days of animal preparation for the experiment. The rectal body temperature was determined with a MicroTherma 2T Hand Held Thermometer (Braintree Scientific, Inc., USA).

Evaluation of the performance of animals was evaluated in a swimming test with a load as described [10]. Cholesterol content (mol/l), triacylglycerides $(\mathrm{mol} / \mathrm{l})$, creatinine $(\mu \mathrm{mol} / \mathrm{L})$, albumin $(\mathrm{g} / \mathrm{l})$, activity of alanine aminotransferase, aspartate aminotransferase $(\mathrm{U} / \mathrm{L})$, gamma glutamyltransferase $(\mathrm{U} / \mathrm{L})$, was determined in the blood serum on a biochemical analyzer Beckman Coulter AU480 (Germany). The content of lipid hydroperoxides in serum was determined as described in [11].

All experiments were repeated no less than in three independent experiments. There were at least 9 animals in each experimental group. As characteristics of the samples obtained mean, standard deviation, standard error of the mean, the sample volume were used. Statistical processing of results was carried out using "Statistica 6" program. The differences between the control and experimental variants were considered as significant at $p \leq 0,05$.

\section{RESULTS}

\section{a. Some Physiological and Biochemical Characteristics of Animals with Cu-Induced Toxicosis}

One of the most significant negative factors inducing disturbances of homeostasis with the further
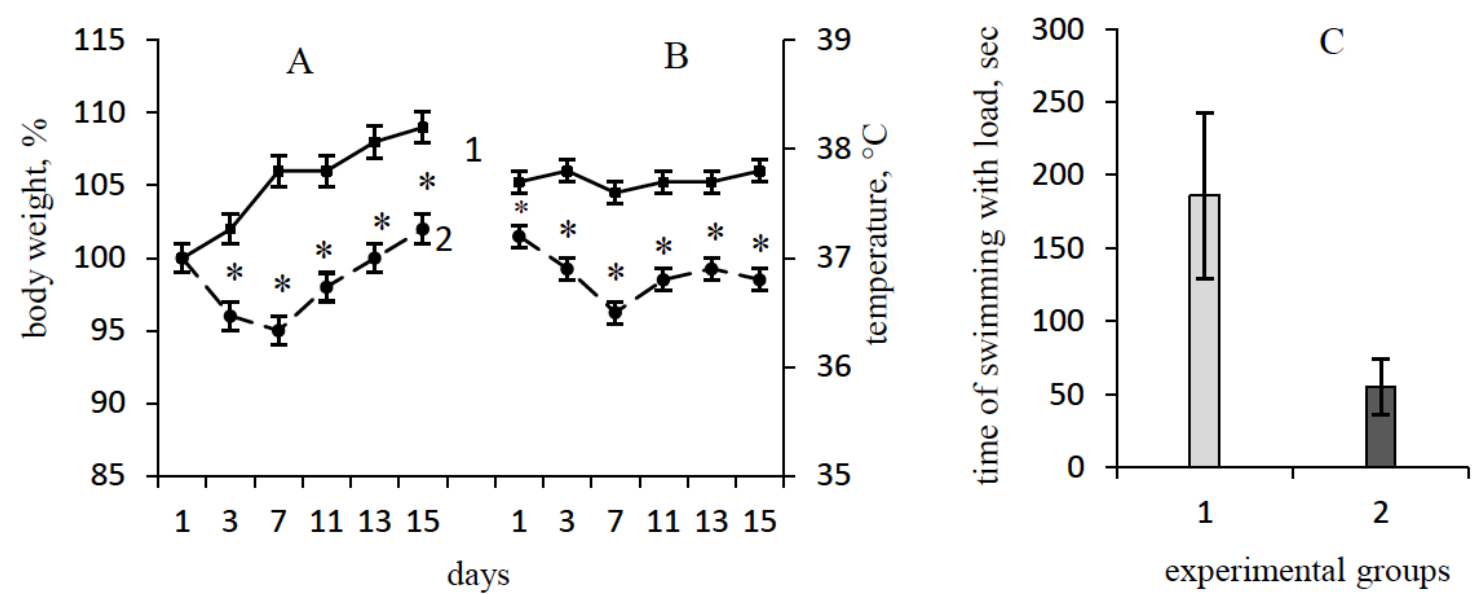

Figure 1: Changes of the body weight $(\mathbf{A})$ and the rectal temperature $(\mathbf{B})$ of the intact group (1) and after the groups after multiple administration of copper sulphate from the 1-st to the 15-th day of observations and the time of swimming with the load (C) of the control (1) and experimental groups after the administration of copper sulphate (2).

* - significant differences between groups $\mathrm{P} \geq 0.05$. 
formation of prepathologies and pathologies are toxic compounds of diverse nature. Common toxicants are heavy metal ions, and in particular copper ions, which are present in significant amounts in water and food [12].

To assess the effect of low-molecular components of the cow colostrum (LMWCC) as a functional feed, the model of $\mathrm{Cu}$-induced toxicosis was used.

Multiple consecutive administrations of $1 \mathrm{mg}$ of copper sulphate per $100 \mathrm{~g}$ of body weight into experimental animals were accompanied by inhibition of the growth rate of animals compared to the animals of the control group (Figure 1A).

On the $3^{\text {rd }}$ day after the beginning of the administration of this toxicant, the mass of animals did not increase in the control group, but decreased by $4 \%$ from the initial body weight. The loss of body weight in such animals lasted until the $7^{\text {th }}$ day of the experiment, and later it was slowly restored to the initial level by the $13^{\text {th }}$ days of observation (Figure 1A).

In experimental animals along with inhibition of body weight growth, the body temperature lowered (Figure 1B). So, if the body temperature of intact animals corresponded to $37.6-37.8^{\circ} \mathrm{C}$ while in animals receiving copper sulphate, it was reduced by 0.8 $1.0^{\circ} \mathrm{C}$ (Figure 1B). Such animals lost the ability to perform swimming with a load (Figure 1C).

Consequently, toxicosis with copper ions, as well as with other toxic compounds, was accompanied by pronounced physiological changes. It is known that the administration of copper ions to experimental animals is accompanied by the accumulation of copper primarily in the liver cells [13]. The increase in copper ions amount in the liver can be accompanied by depression of the liver and the development of the initial stages of liver fibrosis [14].

To assess liver function, the activity of alanine aminotransferase and aspartate aminotransferase was assessed as an indicator of the functional activity of the liver. We report that after two days after the first administration of copper sulphate, the activity of these transferases did not change in comparison with the control level (Figure 2). The amount of cholesterol, triacylglycerols, creatinine and albumin remained unchanged in comparison with the control (Figure 2). The activity of gamma-glutamyltransferase (GGT) increased by $263 \%$. The content of lipid hydroperoxides in blood serum increased by $37 \%$, which correlated with the decrease in the activity of one of the antioxidant enzymes, glutathione peroxidase (Figure 2).

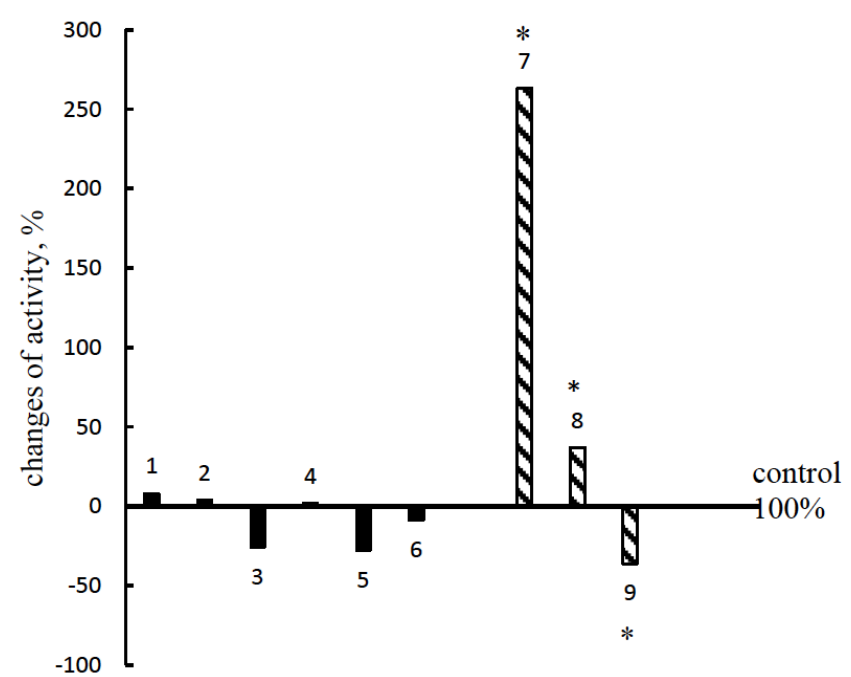

Figure 2: Changes in ALT (1), AST (2), cholesterol (3), triacylglyceride (4), creatinine (5), albumin (6), GGT activity (7), lipid hydroperoxides (8) and glutathione pyroxidase (9) in blood serum in animals after administration of copper sulphate in percent, in relation to the control level, which is taken as $100 \%$.

* - significant differences between groups $\mathrm{P} \geq 0.05$.

Consequently, Cu-induced toxicosis was accompanied by a change of physiological characteristics of animals, while the main functional parameters of the liver remained normal. However, the liver showed oxidative stress (inhibition of the antioxidant enzyme glutathione peroxidase and an increase of the amount of lipid hydroperoxides) and an increase in the activity of gamma-glutamyltransferase. These results suggest the initial stage of liver dysfunction.

Such functional changes in the liver can be induced by various factors: drugs, alcoholic beverages, toxic compounds of diverse nature, and such model can be used to develop functional nutrition concept.

\section{b. Influence of LMWCC on the Physiological and Biochemical Parameters of Experimental Animals with Inhibition of Liver Function}

Three consecutive administrations of LMWCC per os to animals with Cu-induced toxicosis showed a dose-dependent effect on changes in body weight and temperature, as well as on the working capacity (swimming with load). Thus, low LMWCC doses $(0.01 / 100 \mathrm{~g}$ of body weight) eliminate the growth retardation and raise the rectal temperature (Figure 3A, B). 
Administration of LMWCC at $0.1 \mathrm{mg} / 100 \mathrm{~g}$ of body weight had a similar effect on body weight growth and body temperature. However, if the doses of LMWCC were increased to $0.1 \mathrm{mg} / 100 \mathrm{~g}$ of body mass, the effect of "recovery" of intensive growth was less evident, and a dose of $3 \mathrm{mg} / 100 \mathrm{~g}$ of body weight even led to an additional inhibition of growth, and it was even smaller than animals receiving copper sulphate on the 13-15 day of the experiment (Figure $3 \mathbf{A}$ ).

A large dose of LMWCC (3 mg/100 $\mathrm{g}$ of body weight) did not affect the body temperature; it remained lower, compared with the intact control (Figure 3B).

Consequently, the optimal dose of LMWCC to eliminate the effects of $\mathrm{Cu}$-induced toxicosis is 0.01 to $0.1 \mathrm{mg} / 100 \mathrm{~g}$ of body mass. Administration of LMWCC at a dose of $0.1 \mathrm{mg} / 100 \mathrm{~g}$ of weight to animals with $\mathrm{Cu}$-induced toxicosis increased the ability of animals to perform work, although it did not restore it to the control values characteristic of intact animals (Figure 3C).

To study the effect of LMWCC on oxidative stress, which was induced by multiple administrations of copper sulphate to experimental animals, the amount of lipid hydroperoxides (LHP) and serum glutathione peroxidase activity were determined after administration of LMWCC at different doses. It was found that a triple administration of LMWCC at a dose of $0.1 \mathrm{mg} / 100 \mathrm{~g}$ of body mass after the intoxication with copper was accompanied by a decrease in the amount of LHP to the values of intact animals (Figure 4A).

If the dose of LMWCC was increased to $0.5 \mathrm{mg} / 100$ $\mathrm{g}$ of body weight, then the amount of LHP remained unchanged compared to the animals treated with copper sulphate and a large dose $3.0 \mathrm{mg} / 100 \mathrm{~g}$ of body weight even caused an increase of LHP by $56 \%$ compared to animals with copper-induced toxicosis and $215 \%$, if compared with the intact control (Figure 4A).

Consequently, LMWCC, administered to an animal with an increased content of LHP in the blood serum, was accompanied by a bimodal dose-dependent response of the organism. A small dose $(0.1 \mathrm{mg} / 100 \mathrm{~g}$ of weight) of LMWCC reduced their amount, and large doses increased them.

As it was already noted, the increase in the LHP amount correlated with a decrease in the activity of the antioxidant enzyme, GP [14]. This correlation was maintained even after the administration of LMWCC at different doses to experimental animals (Figure 4B).

Thus, the LMWCC at $0.1 \mathrm{mg} / 100 \mathrm{~g}$ of body weight increased the activity of GP compared to intact control
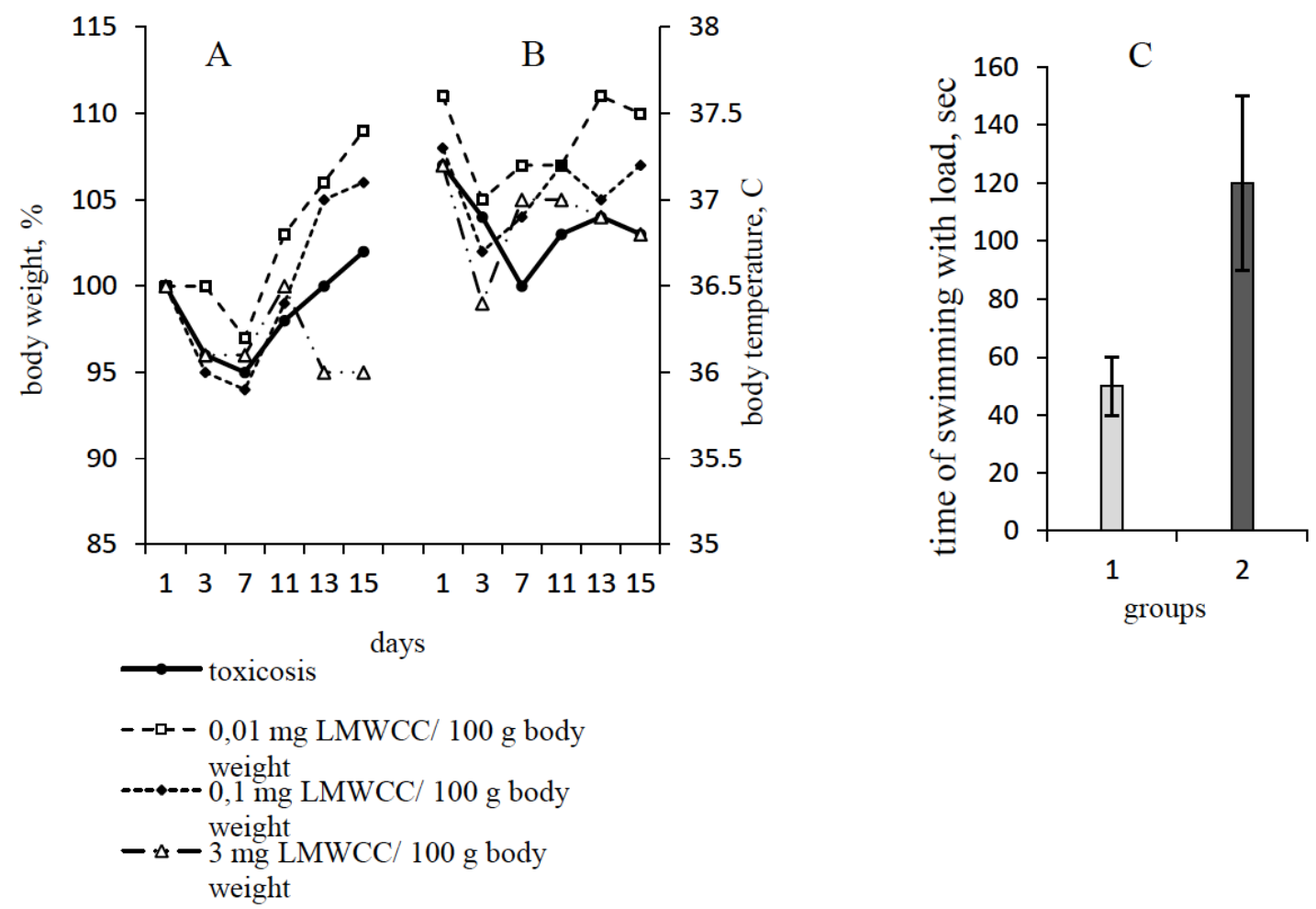

Figure 3: Body weight $(\mathbf{A})$, body temperature $(\mathbf{B})$ and time of swimming with load $(\mathbf{C})$ in a group of animals with Cu-induced toxicosis (1) and after administration of per os LMWCC (2). 


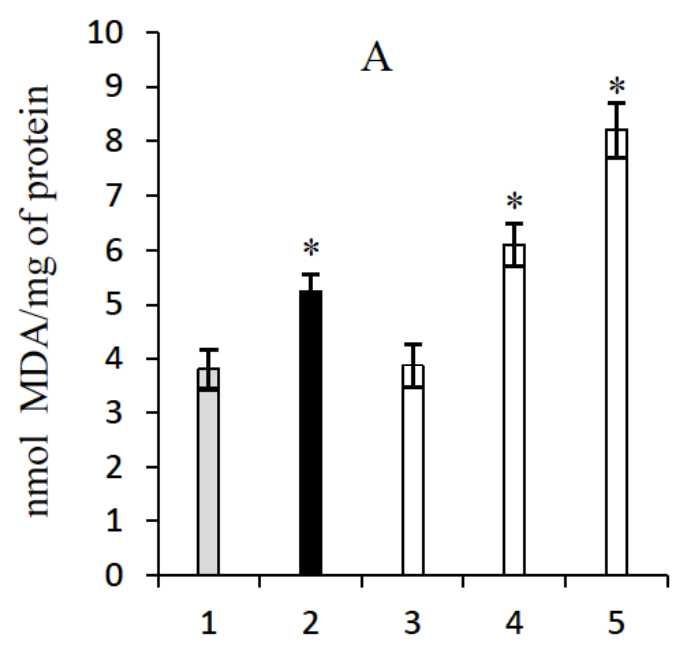

groups

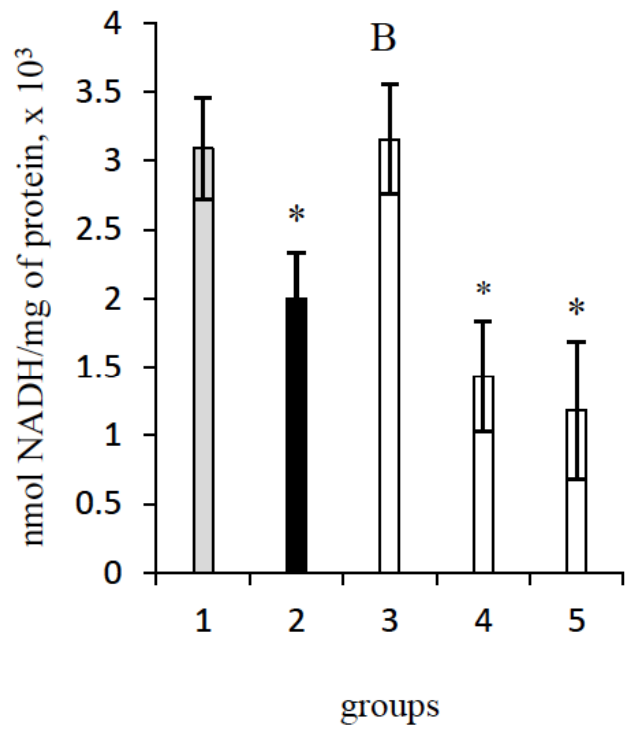

Figure 4: The amount of lipid hydroperoxides $(\mathbf{A})$ and the activity of glutathione pyroxidase (B) in the control group (1), animals after multiple production of copper sulphate (2), and after the production of LMWCC in a dose of $0.1 \mathrm{mg} / 100 \mathrm{~g}(3) \mathrm{ln}$ a dose of $0.5 \mathrm{mg} / 100 \mathrm{~g} \mathrm{(4)}$ and $2.0 \mathrm{mg} / 100 \mathrm{~g} \mathrm{(5)}$ after receiving toxic doses of copper sulphate.

* - significant differences between groups $\mathrm{P} \geq 0.05$.

values, and an increase of the dose to 0.5 and 2 $\mathrm{mg} / 100 \mathrm{~g}$ of body weight was accompanied by a decrease in its activity by $30 \%$ and $41 \%$, respectively, compared with animals receiving the copper sulphate only (Figure 4B).

The amount of cholesterol, triacylglycerides, creatinine, and albumin in the blood serum after administration of LMWCC at different doses to the experimental animals remained unchanged compared with the animals receiving the copper sulphate only.

\section{DISCUSSION}

The general intoxication of the organism is wellknown as such a morbid state that is caused by the prolonged action of exogenous and endogenous toxic compounds of diverse nature [16].

The most common causes of toxicosis may be lowquality products, drugs, chemicals and, in particular, heavy metal ions [17]. Chemotherapy is always accompanied by a pronounced intoxication of the organism [18].

Along with exogenous toxicosis, endogenous toxicosis can also occur. It can arise as a result of deterioration of cell and tissue structures, due to burns, radiation damage, trauma and various autoimmune pathologies [19]. The exogenous toxic factors always induce secondary endotoxic products. It must be taken into account when choosing a strategy for antitoxic activities. The intoxication of the body with copper ions was accompanied by the accumulation of copper ions in various organs and, above all, in the liver. Most of the copper ions were found in the fraction of copperbinding proteins of the cytosol of liver cells, mitochondria and membranes of the endoplasmic reticulum of hepatocytes [20]. Previously, it was shown that three consecutive administrations of copper sulphate ions induced the destruction of hepatocytes, which was accompanied by the synthesis of connective tissue in the liver [21].

Therefore, copper ions, entering the body, serve as both exogenous toxicants and induce the formation of endogenous toxic compounds. Probably, this kind of combination of exogenous and endogenous toxicity occurs not only for Cu-induced toxicosis, but also for toxicosis of another aetiology.

In response to primary toxicosis, which is manifested in the loss of efficiency, weakness, flatulence, diarrhoea and other ailments [22], at least a few strategic options can be used by the organism: elimination of toxicosis factors and restoration of physiological and biochemical indexes to typical levels; transition to a chronic condition with the subsequent formation of various chronic pathologies, in particular, toxic hepatitis, fibrosis, liver cirrhosis, etc.

Currently, the fights against toxicosis are reduced by the following: 1 - elimination of the source of toxic 
compounds; 2 - activation of the excretion of toxic compounds.

At the same time, the most important component in eliminating the negative consequences of toxicoses must be a system of correction of metabolic states aimed at "fixation = remembering" of the alternative metabolic variants, which we classified as chronic pathologies. We are convinced that such correction should be carried out not by farmaceuticals, but by functional food products.

From this point of view, functional nutrition is not so much the food for systematic use in order to reduce the risk of developing diseases and to prevent the deficiency of nutritional ingredients, as the food to eliminate the potentially possible chronic pathologies. This food should be used for limited periods of time, not for systematic nutrition. In this regard, a promising product for functional nutrition can be the cow colostrum.

However, at Cu-induced toxicosis, the activity of alanine transferase and aspartate aminotransferase, cholesterol, triacylglyceride, creatinine and albumin remained at the level of intact control. This indicates that the functional activity of LMWCC 24 hours after the sequential administrations of copper sulphate did not change.

At the same time, the activity of gammaglutamyltransferase, which participates in the exchange of aminoacids, in the liver increased by $250 \%$ compared with the intact control.

As it is known, an increase of the activity of GGT by a dozen times in the blood serum suggests the development of hepatitis, fibrosis and cirrhosis [23]. The relatively small increase in GGT activity, in comparison with the stable activity of ALT and AST, and other functional parameters of the liver, indicates that the scheme of Cu-induced toxicosis used in this work, led to the initial stages of liver function abnormalities. It should be noted that such initial stages of inhibition of liver function can take place in a real situation and can be attributed to pre-pathology, which can be adjusted by food.

As it is known, one of the primary reactions of biological systems to a variety of stress factors is the pro-antioxidant system [24]. This position is supported by the results of this work. Thus, comparatively to the background of the preservation of the functional parameters of the liver as normal, an increase of the amount of lipid hydroperoxides increases and the activity of glutathione peroxidase decreases.

Therefore, one of the "primary" responses of metabolic reactions to toxicosis is the shift in the balance of the prooxidant-antioxidant system towards the prooxidants and the increase in the activity of gamma-glutamyltransferase.

The restoration of glutathione peroxidase activity by LMWCC to the level of intact control correlated with the normalization of serum lipid peroxide hydroperoxides. Restoration of the pro-antioxidant system to the level of intact animals correlated with complete or partial recovery of body weight, body temperature and working capacity. Such results suggest that LMWCC restored metabolic rate on the background of inhibitory action of copper ions.

The mechanism of restoration of the oppressed metabolism cannot be reduced only to the activity of the pro-antioxidant system. The LMWCC are represented by various biologically active compounds that regulate not only the redox system of the organism, but also the activity of the immune system, possibly both the body's detoxification systems and anabolic processes.

An important aspect of the LMWCC effects is the dose-response characteristics of the organism answer to the LMWCC. If small doses of $0.05-0.01 \mathrm{mg} / 100 \mathrm{~g}$ of body weight caused a pronounced antioxidant effect, then large doses of 1 to $3 \mathrm{mg} / 100 \mathrm{~g}$ of body weight, in contrast, had pronounced prooxidant activity. The mechanisms of such activity require special studies. However, it can be assumed that LMWCC contains different compounds that exhibit both pro- and antioxidant activity. Since the ratio of these components is different, then at low concentrations, antioxidants are contained in a larger amount, and as the dose increases, the number of components that induce prooxidant activity increases. When certain concentrations (up to $1 \mathrm{mg} / 100 \mathrm{~g}$ of body weight and more) are achieved, the prooxidant activity exceeds the antioxidant activity. In connection with this, when LMWCC is included in the functional nutrition, it is necessary to adhere strictly to the daily doses of LMWCC.

In conclusion, we note that LMWCC can be included in the composition of functional foods recommended for toxicosis of various aetiologies. 


\section{REFERENCES}

[1] Ingvartsen KL, Moyes KL. Nutrition, immune function and health of dairy cattle. Animal 2013; 7: 112-122. https://doi.org/10.1017/S175173111200170X

[2] Goff JP. Major advances in our understanding of nutritional influences on bovine health. Journal of Dairy Science 2006; 89: 1292-1301.

https://doi.org/10.3168/jds.S0022-0302(06)72197-X

[3] Mathias $\mathrm{R}$, Müller $\mathrm{K}$, et al. Clinical applications of bovine colostrum therapy: a systematic review. Nutrition Rewires 2014; 72: 237-254.

[4] Bagwe SL, Leo JP, et al. Bovine colostrum: an emerging nutraceutical. Journal of Complementary and Integrative Medicine 2015; 12: 175-185. https://doi.org/10.1515/jcim-2014-0039

[5] Conneely MP, Berry DP, et al. Effect of feeding colostrum at different volumes and subsequent number of transition milk feeds on the serum immunoglobulin G concentration and health status of dairy calves. Journal of Dairy Science 2014; 97: 6991-7000. https://doi.org/10.3168/jds.2013-7494

[6] Sanchez-Soto EP, Ponce-Ramos RL, et al. Colostrum proinflammatory cytokines as biomarkers of bovine immune response to bovine tuberculosis (bTB). Microbial Pathogenesis 2017; 103: 57-64. https://doi.org/10.1016/j.micpath.2016.12.007

[7] McGrath BA, Fox PF, et al. Composition and properties of bovine colostrum: a review. Dairy Science \& Technology 2016; 96: 133-158. https://doi.org/10.1007/s13594-015-0258-x

[8] Magistrelli D, Rosi F. Effect of Technological Treatments on Human-Like Leptin Level in Bovine Milk for Human Consumption. Foods 2014; 3: 433-442. https://doi.org/10.3390/foods3030433

[9] Council Directive 86/609/EEC of 24 November 1986 on the approximation of laws, regulations and administrative provisions of the Member States regarding the protection of animals used for experimental and other scientific purposes. Official Journal 1986; L 358: 0001-0028.

[10] Bozhkov Al, Nikitchenko YuV, Klimova EM, Lenkevich ES, Lebed EN, Al-Bakhadly AMM, Alsardia MMA. Young and old animals use various metabolic adaptation strategies to $\mathrm{Cu}$ induced liver fibrosis. Uspeshi gerontologii (in Russian) 2016; 29: $555-566$.

[11] Ohkawa H, Ohahi N, Jadi K. Assay for lipid peroxides in animal tissues by thiobarbituric acid reaction. Anal Biochem 1979; 95: 351-358. https://doi.org/10.1016/0003-2697(79)90738-3

[12] Lim AP, Aris AZ. A review on economically adsorbents on heavy metals removal in water and wastewater. Reviews in Environmental Science and Bio/Technology 2014; 13: 163181.

https://doi.org/10.1007/s11157-013-9330-2
[13]

Bozhkov A, Padalko V, Dlubovskaya V, Menzyanova N Resistance to heavy metal toxicity in organisms under cronic exposure. Indian Journal of Experimental Biology 2010; 48: 679-696.

[14] Bozhkov Al, Klimova EM, Nikitchenko YuV, Davydov VV, Zvyagintseva OV, Kurguzova NI. Sidorov VI, Naglov AV. Stem cells take part in regulation of prooxidant activity and immunity at liver fibrosis. American Journal of Biomedical and Life Sciences: Special Issue: Mechanisms of Protection Against Oxidative Stress 2014; 2: 5-12.

[15] Kurguzova NI, Bozhkov Al, Nikitchenko YV, Al Begai MAY, Goltvyansky AV, Alsardia MMA, Bozhkov AA. Interconnection of Antitoxic and Antioxidant Systems of the Organism under the Action of Natural Low Molecular Complex - Fungidol. American Journal of Biomedical and Life Sciences. Special Issue: Mechanisms of Protection against Oxidative Stress 2015; 2: 25-32.

[16] Schou M. Biology and pharmacology of the lithium ion. Pharmacological Reviews March 1957; 9: 17-58.

[17] Jan AT, Azam M, Siddiqui K, Ali A, Choi I, Haq QMR. Heavy Metals and Human Health: Mechanistic Insight into Toxicity and Counter Defense System of Antioxidants. Int J Mol Sci 2015; 16: 29592-29630.

https://doi.org/10.3390/ijms161226183

[18] Panahi Y, Ghanei M, Vahedi E, Ghazvini A, Parvin S Madanchi $\mathrm{N}$, et al. Effect of recombinant human IFNy in the treatment of chronic pulmonary complications due to sulfur mustard intoxication. J Immunotoxicol 2014; 11: 72-7. https://doi.org/10.3109/1547691X.2013.797525

[19] Pollard KM, Hultman P, Kono DH. Toxicology of Autoimmune Diseases. Chem Res Toxicol 2010; 15: 455-466. https://doi.org/10.1021/tx9003787

[20] Bozhkov Al, Sidorov VI, Kurguzova NI, Dlubovskaya VL. Metabolic memory enhances the effect of hormesis on copper ions and is of an age-dependent character.Uspeshi gerontologii (in Russian) 2014; 27: 72-80.

[21] Bozhkov Al, Linkevych OS, Ivanov EG, Klimova OM, AIBegai MAY. Low molecular weight components of colostrum regulate the activity of cellular component of the immune system in animals with $\mathrm{Cu}$-induced liver fibrosis. International Journal of Current Research 2016; 8: 44129-44137.

[22] Baghdadi HH, El-Demerdash FM, Radwan EH. The protective effect of Coriandrum sativum L. oil against liver toxicity induced by Ibuprofen in rats. Journal of Bioscience and Applied Research 2016; 2: 197-202.

[23] Omer SAM. Study activity of serum gamma glutamyl transferase enzyme as a diagnostic biomarker in alcoholic hepatitis. Asian Journal of Biomedical and Pharmaceutical Sciences 2016; 6: 01-02.

[24] Bozhkov AI, Nikitchenko YuV, Sheremet AA, Bozhkov AA The prooxidant-antioxidant system is the primary stage of the answer of organism on unbalanced diet. Journal of Harmonized Research in Pharmacy 2014; 3: 78-90. 